\title{
Decoupling of regional neural activity and inter- regional functional connectivity in Alzheimer's Disease - a simultaneous PET/MRI study
}

\section{Somayeh Maleki Balajoo}

Forschungszentrum Jülich: Forschungszentrum Julich $\mathrm{GmbH}$

\section{Farzaneh Rahmani}

University of Washington School of Medicine

\section{Reza Khosrowabadi}

Shahid Beheshti University

\section{Chun Meng}

University of Electronic Science and Technology of China

\section{Simon B. Eickhoff}

Forschungszentrum Julich ICG: Forschungszentrum Julich GmbH

\section{Timo Grimmer}

TU München: Technische Universitat Munchen

\section{Mojtaba Zarei}

Shahid Beheshti University

\section{Alexander Drzezga \\ Uniklinik Köln: Uniklinik Koln}

\section{Christian Sorg}

TU München: Technische Universitat Munchen

Masoud Tahmasian ( $\sim$ m.tahmasian@fz-juelich.de)

Forschungszentrum Jülich: Forschungszentrum Julich GmbH https://orcid.org/0000-0003-3999-3807

\section{Research Article}

Keywords: Alzheimer's disease, Mild cognitive impairment, PET/MR, Neural activity, Functional connectivity, Graph analysis

Posted Date: August 23rd, 2021

DOl: https://doi.org/10.21203/rs.3.rs-823388/v1

License: (c) (1) This work is licensed under a Creative Commons Attribution 4.0 International License. Read Full License 


\section{Abstract}

Purpose: Alzheimer's disease (AD) and mild cognitive impairment (MCI), a syndrome at-risk for $A D$, are characterized by both aberrant regional neural activity and disrupted inter-regional functional connectivity (FC). It is, however, not clear how aberrant regional neural activity and inter-regional FC interact across $\mathrm{MCl}$ and $\mathrm{AD}$. Thus, we investigated the interplay between regional neural activity and inter-regional topological measures of $\mathrm{FC}$ in $\mathrm{MCl}$ and $\mathrm{AD}$ using simultaneous PET/MR measurement.

Methods: We scanned 19 patients with $\mathrm{MCl}, 33$ patients with $\mathrm{AD}$, and 26 healthy individuals by simultaneous FDG-PET/resting-state fMRI. First, we assessed regional glucose metabolism identified through FDG-PET (as a proxy of regional neural activity), and inter-regional FC topology through clustering coefficient and degree centrality (as surrogates of local segregation and global connectivity, respectively, based on fMRI blood oxygenation). Next, we examined the potential moderating effect of disease status ( $\mathrm{MCl}$ or $\mathrm{AD}$ ) on the link between regional metabolism and inter-regional $\mathrm{FC}$ topology using hierarchical moderated multiple regression analysis.

Results: Not only regional metabolism and inter-regional FC metrics were disrupted in in $\mathrm{MCl}$ and $\mathrm{AD}$ patients, but also AD significantly alters coupling between regional metabolism and inter-regional FC, particularly in the right inferior temporal, supplementary motor area and planum temporal areas, where $A D$ moderated the effect of their regional glucose metabolism on predicting their inter-reginal FC.

Conclusion: Our findings demonstrated that $A D$ decouples the association between regional neural activity and functional segregation.

\section{Introduction}

Alzheimer's disease (AD) and mild cognitive impairment $(\mathrm{MCl})$ are characterized by distinct patterns of neuronal loss leading to reduced overall neural activity, which can be indirectly quantified through fluorodeoxyglucose-positron emission tomography (FDG-PET) [1]. Moreover, regional glucose metabolism $(\mathrm{rFDG})$ is associated with cortical atrophy and beta-amyloid/tau protein accumulation, which are hallmarks of AD pathology [2,3]. Functional connectivity (FC) is quantified as the magnitude of functional co-activation between neural activity in distinct brain regions, typically measured by restingstate functional magnetic resonance imaging (rs-fMRI). Several studies demonstrated FC alterations, mainly in the default mode network (DMN), along the trajectory of $A D$ [4-6]. Topological features of these FC networks can be modeled through graph theory analyses, in which brain regions are considered as "nodes", and FC between them as "edges" of the graph [7]. Clustering coefficient (CC) and degree centrality (DC) are two commonly used inter-regional FC topological metrics, representing local segregation and centrality of individual nodes, respectively [8]. In particular, CC quantifies how well a certain node has formed locally-segregated processing modules (i.e., clusters) around itself, while DC gives a measure of the importance/centrality of a node in terms of interacting with other nodes within 
and outside the regional clusters or how well it is contributing to regional segregation and global integration of the network at the same time [7].

As $80 \%$ of neural metabolic activity is dedicated to synaptic signaling [9], FC might indicate a conjugate in-/decreased blood oxygenation level-dependent (BOLD) signaling between the brain regions. Broadly speaking, empirical evidence and computational modelling [10-12] suggest that higher local metabolism (as a proxy for local activity) in a particular brain region increases its sensitivity to afferent input from other regions and thus determines the likelihood of inter-regional FC (as a proxy for synchronous fluctuations of BOLD signal between regions). A recent study applied simultaneous functional FDG$\mathrm{PET} / \mathrm{rs}-\mathrm{fMRI}$, provided evidence for synchrony and interaction between the neural dynamics of glucose metabolism and the BOLD hemodynamic response [13]. These findings together suggest strong coupling between glucose metabolism and FC in healthy brains. In healthy brains, up to $18 \%$ of inter-subjects variability in whole-brain glucose metabolism can be explained by differences in FC [14]. Moreover, it has been shown that increased FC and higher functional clustering are associated with a non-linear increase in regional metabolism in healthy individuals [14]. This association, however, could be disturbed in AD, where rFDG no longer linked with regional functional activity in target $A D$ regions in the posterior associational cortices [15]. It has been suggested that local amyloid beta pathology might be responsible for this decoupling (i.e., loss of correlation) between rFDG and inter-regional FC in the default mode network (DMN) in AD patients [16]. However, how aberrant regional neural activity and inter-regional FC interact in $\mathrm{MCl} / \mathrm{AD}$, and how $\mathrm{AD}$ (we refer to it as a clinical diagnosis), affect the link between rFDG and FC topological characteristics are poorly understood. Here, we aimed to address these questions using simultaneous acquisition of FDG-PET and rs-fMRI in healthy controls $(\mathrm{HC})$ and patients with $\mathrm{MCl}$ and $\mathrm{AD}$.

\section{Methods}

\subsection{Participants}

Thirty-three patients with mild AD-dementia, 19 patients with $\mathrm{MCl}$ and $26 \mathrm{HC}$ subjects were recruited in this cross-sectional study. Patients were randomly selected from outpatient memory clinic of the Department of Psychiatry and Psychotherapy of Klinikum rechts der Isar, School of Medicine, Technical University of Munich (TUM). Diagnosis of $A D$ or $\mathrm{MCl}$ was determined using Clinical Dementia Rating (CDR) and neuropsychological testing batteries based on criteria established by Consortium to Establish a Registry for Alzheimer's disease CERAD. Twenty-six HCs were also recruited through word-of-mouth advertising in Munich. Demographic information is provided in Table 1. Importantly, as our AD/MCl patients did not have amyloid/tau PET imaging, use of "effect of $A D$ " phrase in this study does not directly correspond to the pathology of disease and merely refers to the presence or absence of clinical diagnosis of MCl/AD. Of note, this study was approved by the TUM ethics committee in line with the institute's Human Research Committee guidelines and conformed to standards of the declaration of Helsinki. Written informed consent was obtained from all participants after providing detailed information about this study. 
Table 1. Demographic and clinical data of participants

\begin{tabular}{|lllll|}
\hline & $\mathrm{HC}(\mathrm{n}=26)$ & $\mathrm{MCl}(\mathrm{n}=19)$ & $\mathrm{AD}(\mathrm{n}=33)$ & $\mathrm{p}$-Value \\
\hline Age, year & & & & $<0.001$ \\
\hline Sex (female) & 9 & 12 & 14 & 0.157 \\
\hline MMSE score & & & $<0.001$ \\
\hline CREAD NAB total & & & $<0.001$ \\
\hline
\end{tabular}

Abbreviations: AD: Alzheimer disease; HC: healthy control; MCl: mild cognitive impairment; MMSE: MiniMental State Examination; CERAD: Consortium to Establish a Registry for Alzheimer's Disease neuropsychological assessment battery. Analysis of variance, $p<0.05$ as threshold of significance, except of sex (Kruskal-Walis test).

\subsection{Data acquisition and preprocessing}

Imaging data acquisition included structural MRI (T1 weighted), rs-fMRI, and FDG-PET, which were simultaneously acquired on a Biograph hybrid PET/MR scanner (Siemens, Erlangen, Germany), processed, and analyzed according to previously published and standardized protocols (Supplemental Methods, Sect. 1) $[6,17,18]$.

\subsection{FDG-PET data analysis}

The mean glucose uptake values of all voxels within the 112 regions of interest (ROIs), defined based on Harvard-0xford atlas [19], were extracted from each subject-specific's preprocessed partial volume corrected PET images. We then normalized the mean glucose uptake value of each ROI by dividing it to the whole-brain glucose uptake value of the same subject, as suggested previously [20].

\section{4. rs-fMRI data analysis}

Mean BOLD signal of all 112 cortical and subcortical ROls were extracted and the inter-regional FC patterns were computed and modeled based on graph analysis, where the ROIs considered as nodes and FC between them (using absolute values of Pearson's correlation coefficients) as edges. Finally, a $112 \times 112$ symmetric undirected weighted matrix representing individual whole brain inter-regional FC was computed for each subject. From a topological perspective, each subject has a different topology in terms of connection density of brain regions. The connection density in a graph is defined as a ratio of number of existing edges to all possible edges [21]. Difference in connection density, in turn, influences most of the extracted topological metrics within a graph [22]. Thus, it is necessary to implement a matching strategy between FC graphs, prior to statistical analyses between the three groups [23]. Accordingly, we thresholded adjacency matrix of each subject for density range from $0.01-0.40$ (with intervals of 0.01 ), as suggested earlier [24]. It means that the adjacency matrix of each subject was thresholded 40 times with different connection densities to ensure that the topology variability across all subjects were considered in the analysis. We then characterized the organization of brain regions in terms 
of CC (segregated role of node) [25] and DC (centralized role of node) [26] (Supplemental Methods, Sect. 2). To assess the inter-regional FC topological metrics, we calculated those metrics for each of 40 thresholded adjacency matrices using the Brain Connectivity Toolbox [7]. Finally, the integral values of CC and DC across the 40 thresholded adjacency matrices for each subject were used for group comparison, as recommended previously [27].

\subsection{Group comparison and statistical analyses}

Group comparisons based on Analysis of covariance (ANCOVA) on rFDG, CC, and DC were performed for all 112 ROIs, while age and sex were considered as the covariates of no-interest. The results were corrected for multiple comparisons using N-region statistical comparison, as described previously [28]. The significance threshold was calculated using $1 /$ number of ROls, as: $1 / 112=0.009$. Post-hoc analyses were then conducted for rFDG, CC and DC utilizing permutation test with 100,000 permutations and significance threshold for post-hoc test was calculated using $p$-value $=1 /$ number of tests to correct for potential false-positive errors.

\subsection{Cross-modality analysis}

To evaluate the moderating role of disease on the link between rFDG the inter-regional FC topology (i.e., $\mathrm{CC} / \mathrm{DC}$ ), we first focused on the brain areas in which rFDG and CC/DC were affected by disease in all group (i.e., overlapping regions), hypothesizing a significant moderating effect of $A D$ on their association. Secondly, we explored the areas in which only one measure (i.e., either rFDG or CC/DC) was affected by disease (i.e., non-overlapping regions). Thus, the potential moderating effect of $\mathrm{MCl}$ or $\mathrm{AD}$ on the link between rFDG and CC/DC was examined using hierarchical moderated multiple regression (HMMR) analysis (Supplemental Methods, Sect. 3; SI-Figure 1) on both overlapping and non-overlapping brain regions to test whether and how clinical diagnosis and rFDG predict CC/DC. To do so, rFDG and clinical groups (i.e., $\mathrm{HC}, \mathrm{MCl}, \mathrm{AD}$ ) were entered as main predictor variables to a model to predict CC/DC as response variable. Then, the interaction as the product term of the main predictor variables " rFDG $\times$ clinical diagnosis " were added to the prediction model. Of note, we removed the effect of age and sex from the response variables, before applying the HMMR analyses. To evaluate whether the interaction between predictor variables ("rFDG $\times$ clinical diagnosis ") is meaningful; we tested particularly whether adding the interaction term increased the variance explained by the model in successive regression steps $\left(\Delta R^{2}\right)$. When a statistically significant interaction emerged, interaction was interpreted according to the available guidelines $[29,30]$. These results were also corrected for multiple comparisons using family wise error (FWE) rate. Moreover, we performed additional control analysis to evaluate whether clinical diagnosis and CC/DC could predict rFDG in both overlapping and non-overlapping regions (Supplemental Methods, Sect. 4).

\section{Results}

\subsection{Regional glucose metabolism alterations along the trajectory of disease}


Comparing rFDG between the three groups (controlling for age and sex) revealed significant reduced metabolism in various regions including the bilateral middle and inferior temporal gyri (ITG), bilateral angular and bilateral lateral occipital gyri in $\mathrm{MCl}$ patients compared to $\mathrm{HC}$ and in $\mathrm{AD}$ patients compared to $\mathrm{MCl}$ (Fig. 1, SI-Table 1). We also found relative hypermetabolism in the bilateral precentral, bilateral parahippocampal, bilateral lingual, bilateral occipital fusiform gyri, bilateral brain stem and right hippocampal gyri in $\mathrm{AD}$ patients compared to both $\mathrm{MCl}$ and $\mathrm{HC}$ groups. These results indicated a pattern of progressive hypometabolism in the bilateral middle and inferior temporal, angular and lateral occipital gyri along the trajectory of disease (Fig. 1 and SI-Table 1).

\subsection{Disrupted local FC network topology along the trajectory of disease}

Like rFDG findings, we observed widespread alterations in CC/DC between groups (Fig. 2, SI-Tables 2 and 3). In particular, CC was more extensively affected than DC in both patients' groups compared to HCs in various cortical regions including bilateral middle and inferior frontal, bilateral superior and inferior temporal, bilateral supramarginal, bilateral lateral occipital, bilateral supplementary motor, bilateral occipital, and bilateral Heschel, and left angular gyri (Fig. 2A, SI-Table 2). Meanwhile, in patients DC was decreased in bilateral precentral, lateral occipital, bilateral Heschel gyri, and increased in bilateral parahippocampal gyri (Fig. 2B, SI-Table 3).

Table 2. Overlapping brain regions determined by the direction of change in regional glucose metabolism and inter-regional functional connectivity topology metrics (CC: clustering coefficient, DC: degree centrality) 


\begin{tabular}{|c|c|c|c|c|}
\hline & \multirow[t]{2}{*}{ Brain regions } & \multirow{2}{*}{$\begin{array}{l}\text { Regional glucose } \\
\text { metabolism } \\
\text { (rFDG) }\end{array}$} & \multicolumn{2}{|c|}{$\begin{array}{l}\text { Inter-regional functional } \\
\text { connectivity topology metrics }\end{array}$} \\
\hline & & & $\mathrm{CC}$ & DC \\
\hline \multicolumn{5}{|l|}{$\begin{array}{l}\mathrm{HC} \\
\text { vs. } \\
\text { MCl }\end{array}$} \\
\hline \multirow{13}{*}{$\begin{array}{l}\text { HC } \\
\text { vs. } \\
\text { AD }\end{array}$} & Precentral Gyrus (L) & $\mathrm{HC}<\mathrm{AD}$ & $H C>A D$ & $H C>A D$ \\
\hline & Precentral Gyrus (R) & $\mathrm{HC}<\mathrm{AD}$ & $H C>A D$ & $H C>A D$ \\
\hline & $\begin{array}{l}\text { *Lateral Occipital Cortex, inferior } \\
\text { division }(L)\end{array}$ & $H C>A D$ & - & $H C>A D$ \\
\hline & $\begin{array}{l}\text { *Parahippocampal Gyrus, } \\
\text { posterior division }(\mathrm{L})\end{array}$ & $H C<A D$ & - & $H C<A D$ \\
\hline & *Brain-Stem (R) & $H C<A D$ & - & $H C<A D$ \\
\hline & $\begin{array}{l}\text { *Superior Temporal Gyrus, } \\
\text { posterior division }(L)\end{array}$ & $\mathrm{HC}>\mathrm{AD}$ & $H C>A D$ & - \\
\hline & $\begin{array}{l}\text { *Inferior Temporal Gyrus, } \\
\text { temporooccipital part (L) }\end{array}$ & $H C>A D$ & $H C>A D$ & - \\
\hline & $\begin{array}{l}\text { *Inferior Temporal Gyrus, } \\
\text { temporooccipital part (R) }\end{array}$ & $H C>A D$ & $H C>A D$ & - \\
\hline & *Superior Parietal Lobule (L) & $H C>A D$ & $H C>A D$ & - \\
\hline & *Angular Gyrus (L) & $H C>A D$ & $H C>A D$ & - \\
\hline & $\begin{array}{l}\text { *Lateral Occipital Cortex, } \\
\text { superior division }(\mathrm{R})\end{array}$ & $H C>A D$ & $H C>A D$ & - \\
\hline & $\begin{array}{l}\text { Temporal Occipital Fusiform } \\
\text { Cortex (R) }\end{array}$ & $H C<A D$ & $H C>A D$ & - \\
\hline & Occipital Fusiform Gyrus (R) & $H C<A D$ & $H C>A D$ & - \\
\hline \multirow{3}{*}{$\begin{array}{l}\mathrm{MCl} \\
\text { vs. } \\
\text { AD }\end{array}$} & Precentral Gyrus (L) & $M C l<A D$ & - & $\mathrm{MCl}>\mathrm{AD}$ \\
\hline & *Brain-Stem (L) & $M C l<A D$ & - & $M C l<A D$ \\
\hline & *Angular Gyrus (L) & $M C l>A D$ & $\mathrm{MCl}>\mathrm{AD}$ & - \\
\hline
\end{tabular}

* The direction of group differences in regional glucose metabolism and inter-regional functional connectivity topology metrics is congruent. 
AD: Alzheimer disease; HC: healthy control; MCl: mild cognitive impairment; L: left; R: right; N.S: Not significant.

\subsection{The moderator effect of $A D$ on the link between regional glucose metabolism and inter-regional FC topological metrics}

\section{The overlapping regions}

To investigate the potential effects of $A D$ on the association between rFDG and CC/DC, we first considered regions where changes in rFDG and CC/DC overlapped in all group comparisons. As there were no overlapping regions in all group comparison pairs including $A D$ vs. $H C, A D$ vs. $M C l$, and $M C l$ vs. $\mathrm{HC}$, we identified overlapping brain regions where changes in FFDG and CC/DC happened simultaneously in each group comparison (Fig. 3, Table 2). Hypometabolism was observed along with a reduction in CC in bilateral temporo-occipital parts of the ITG, right superior temporal gyrus, right lateral occipital cortex, left angular gyrus and left superior parietal lobule, while relative hypermetabolism was associated with reduced $\mathrm{CC}$ or $\mathrm{DC}$ topological metrics in the right temporal occipital fusiform and occipital fusiform cortices, and bilateral precentral gyri in $\mathrm{AD}$ or $\mathrm{MCl}$ groups compared to $\mathrm{HCs}$. There was also an increase in both regional metabolism and $D C$ in the right brain stem $(A D>H C)$ and the left brainstem $(A D>M C I)$.

Next, we applied the HMMR model to predict CC/DC by exploring the interaction effect between rFDG and clinical diagnosis, while correcting for multiple comparisons using FWE correction ( $p$-value $<0.05$ / number of all interaction tests for the overlap regions $=(0.05 / 18)<=0.0027)$. There was a significant two-way interaction between "rFDG $\times$ clinical diagnosis" in the right ITG that predicted changes in CC, when comparing $\mathrm{HC}$ and $\mathrm{AD}$ groups $\left(\mathrm{R}^{2}=0.4, \mathrm{~F}(4,55)=12, \mathrm{p}_{(\text {model })}<0.00001\right)$. Adding the interaction term $\left(\mathrm{rFDG} \times\right.$ clinical diagnosis), significantly improved the model in the right ITG $\left(\Delta \mathrm{R}^{2}=0.08, \mathrm{p}_{\text {interaction }}=\right.$ 0.008) (Fig. 4A). No significant interaction between rFDG and clinical diagnosis predicted CC laterations, when comparing $A D$ vs. $\mathrm{MCl}$ or $\mathrm{MCl}$ vs. $\mathrm{HC}$ groups. In addition, the interaction between rFDG and clinical diagnosis did not predict DC alterations in any of group comparisons. In the right ITG, CC showed a negative association with rFDG in $\mathrm{HC}$ subjects, but a positive association in $\mathrm{AD}$ patients. The significant interaction between rFDG and clinical diagnosis indicated that the local properties of right ITG (CC and rFDG) depends on clinical diagnosis.

\section{The non-overlapping regions}

Using the same HMMR model in the non-overlapping regions, there was a significant interaction between "rFDG $\times$ clinical diagnosis" in the right supplementary motor area (SMA) which predicted CC alterations between $A D$ and HC groups $\left(F(4,55)=23.9, p_{\text {(model) }}<0.0001\right.$; Supplemental Results, Sect. 1) (Fig. 4B). Moreover, there was a significant interaction between "rFDG $\times$ clinical diagnosis" in the right planum temporale that was able to predict DC changes $\left(F(4,55)=7.94, \mathrm{p}_{\text {(model) }}=0.00017\right.$; Supplemental Results, Sect. 1) (Fig. 4C). 


\subsubsection{The findings of control analysis in both overlapping and non-overlapping regions}

Finally, we performed a control analysis to assess in which brain regions the interaction between CC/DC and clinical diagnosis could predict rFDG alterations. This analysis yielded to no significant result for any of the overlapping regions. In the non-overlapping regions, however, the analysis revealed that a two-way interaction between "CC $\times$ clinical diagnosis" in the left middle temporal gyrus $\left(R^{2}=0.58, F(5,53)=25.1\right.$, $\left.\mathrm{p}_{\text {(model) }}<0.0001\right)$, the left anterior supramarginal gyrus $\left(\mathrm{R}^{2}=0.34, \mathrm{~F}(4,55)=9.45, \mathrm{p}_{\text {(model) }}<0.0001\right)$ and the left posterior supramarginal gyrus $\left(R^{2}=0.53, F(4,55)=20.7, p_{\text {(model) }}<0.0001\right)$ were able to predict alterations in rFDG between HC and AD groups (Supplemental Results, Sect. 2; SI-Figure 2). Moreover, our findings showed a two-way interaction between " $D C \times$ clinical diagnosis" in the left middle temporal gyrus (temporooccipital part) $\left(R^{2}=0.6, F(4,55)=27.9, p_{\text {(model) }}<0.0001\right)$, the left posterior supramarginal gyrus $\left(R^{2}=0.55, F(4,55)=22.9, p_{(\text {model })}<0.0001\right)$ and the left posterior parahippocampal gyrus $\left(R^{2}=0.3, F\right.$ $\left.(4,55)=7.88, \mathrm{p}_{\text {(model) }}=0.0002\right)$ that predict $\mathrm{rFDG}$ alterations between the $\mathrm{AD}$ and $\mathrm{HC}$ groups (Supplemental Results, Sect. 2; SI-Figure 3).

\section{Discussion}

We examined the association between alterations in regional neural activity and topological measures of inter-regional $\mathrm{FC}$ and their interactions in $\mathrm{HC}, \mathrm{MCl}$, and $\mathrm{AD}$. Our findings demonstrated the following points: i) cortical hypometabolism along with reduced CC/DC in various brain regions (Fig. 1, 2); ii) congruent alterations in rFDG and FC topology in form of reduced rFDG and reduced CC/DC in the bilateral inferior temporal gyri and left superior temporal gyrus, left superior parietal lobule, left angular gyrus and left lateral occipital gyrus (Fig. 3); iii) incongruent alterations in form of increased rFDG associated with reduced CC/DC in the right temporal occipital fusiform and bilateral precentral cortices (Fig. 3); iv) a significant two-way interaction between rFDG and "clinical diagnosis as AD" predicted alterations in CC of the right ITG among the overlapping regions (Fig. 4A); v) a significant two-way interaction between rFDG and "clinical diagnosis i.e., $A D$ " predicted $C C$ alterations in the right SMA and $\mathrm{DC}$ alterations in the right planum temporale among the non-overlapping regions (Figs. 4B, 4C); and vi) a significant two-way interaction between inter-regional FC metrics and "clinical diagnosis i.e., AD" predicted rFDG alterations in the left middle temporal, supramarginal, and parahippocampal, among the non-overlapping regions in the control analysis (SI-Figures 2,3). These findings suggest that AD disrupts a coupling between rFDG and FC topology in the right inferior temporal and supplementary motor area, as well as the left middle temporal, supramarginal, and parahippocampal regions.

\subsection{Hypometabolism and disrupted inter-regional FC topology in $\mathrm{MCl} / \mathrm{AD}$}


Hypometabolism and FC disruption, mainly in the posterior part of DMN, are well-documented features of $A D$ that are suggested to be strongly linked with grey matter atrophy, amyloid beta deposition and tau accumulation $[3,18,31]$. We also observed relative hypermetabolism in $\mathrm{MCl}$ and $\mathrm{AD}$ in the bilateral precentral and lingual gyri, in line with previous studies [6,32,33]. Moreover, changes in the topology of the functional connectivity networks of several cortical areas within and outside the DMN have been reported in $\mathrm{MCl}$ and $\mathrm{AD}$ [34]. Neurobiological mechanisms of the link between regional changes of metabolism and disruption of functional network topology is yet to be investigated. Of note, it has been suggested that functional decoupling between the temporal lobe and posteromedial cortex, as the main hubs of DMN, contributes to disinhibition-like changes in the hippocampus and induce hyperexcitability in the medial temporal lobe [5]. This pathological hypermetabolism in the medial temporal lobe might precipitates the age-associated tau deposition in this region, leading to a loss of white matter integrity and structural dysconnectivity between the temporal lobe and posteromedial cortex [35].

\subsection{The effect of "clinical diagnosis as $A D$ " on the reginal neural activity and inter-regional FC topology link}

We revealed that $A D$ altered the coupling between rFDG and inter-regional FC topology matrices. In particular, $A D$ interacts with IFDG to predict regional $C C$ of the right ITG and right SMA and to predict DC in the right planum temporale. Aberrant coupling between rFDG and $\mathrm{FC}$ topology metrics in patients with $A D$ indicates that $A D$ might affects the rFDG-CC and rFDG-DC links. The altered association between rFDG and FC in AD has been well-documented previously. For example, A longitudinal study demonstrated that severity of amyloid deposition in the regions with high degree of amyloid beta pathology (i.e., in the posterior $\mathrm{DMN}$ ) can predict progressive hypometabolism in remote, but functionally connected areas, with minimal amyloid pathology [36]. In another study, rFDG/FC decoupling was found in several areas within the posterior DMN [15], followed by another study demonstrating that the rFDG/FC decoupling in the posterior DMN is directly correlated with amyloid beta deposition [16]. In this study, Scherr and colleagues found that rFDG progressively decoupled from FC in the posterior DMN and degree of this decoupling associated with amyloid beta load [16]. The authors identified "rFDG-FC coupling" as the only significant variable, which predicted cognitive status of the patients with early and late $\mathrm{MCl}$ and AD. Our results support their findings by showing the adverse effects of AD on coupling between rFDG and topological metrics of inter-regional FC. Similar to this study [16], we found widespread and congruent alterations in $\mathrm{FFDG}$ and $\mathrm{FC}$ topology in the main hubs of DMN, including the parahippocampal gyrus, and the middle and inferior temporal gyri, suggesting that $A D$ pathology in the $D M N$ can be (at least partly) accountable for the changes observed in our study. It is worthy to mention that amyloid beta and tau pathology interact in their regulation of synaptic function. Synaptic tau and amyloid deposition mutually precipitate signaling pathways that culminate in progressive synaptic dysfunction and loss [37, 38]. Indeed, it has been demonstrated that in individuals with normal amyloid-beta level, FC has an inverse correlation with the degree of tau deposition [39]. A similar model demonstrated that cascading network failure is mediated by amyloid deposition in the DMN along with global tau deposition [40]. Based on these findings and a recently proposed model by our group [5], it seems that amyloid beta and 
tau pathology might be important driving forces for alterations in rFDG, FC topology, and rFDG/FC decoupling in $A D$, which should be directly tested in future.

The role of ITG in AD has been assessed and it's hypometabolism, despite minimal amyloid beta deposition is reported earlier [41]. Interestingly, hypometabolism in the ITG is linked with conversion from $\mathrm{MCl}$ to $A D$, which is known to be associated with increased whole brain amyloid burden [42, 43]. Similarly, higher activity in the ITG predicts better cognitive reserve in AD patients [44, 45]. Importantly, amyloid beta deposition in the parieto-temporal cortices is associated with longitudinal tau deposition in the ITG in early $A D$ [46], suggesting that tau pathology might be an important contributor to rFDG/CC decoupling in the ITG. In addition, we identified that interaction between changes in rFDG and clinical diagnosis predicted the $\mathrm{DC}$ alterations in the planum temporale. The planum temporale is an important subcomponent of the superior temporal gyrus and auditory cortices and is highly asymmetric between left and right hemispheres primarily as a reflection of language lateralization [47], but also as a result of handedness [48]. This asymmetry in cortical thickness and morphology becomes more pronounced in AD, as evidenced by post-mortem findings of altered pyramidal cortex morphology in this region [49]. Finally, increased amyloid burden in post-mortem brains of $A D$ patients in this region have been shown to be associated with cognitive decline [50].

Although intact glucose metabolism in the primary motor cortex (M1) and SMA has been considered as a distinguishing feature of $A D$ from other dementias [51, 52], it has been suggested that hypermetabolism in some cortical regions occurring in the early stage of disease may represent a compensatory response to the neural damage [33]. Here, we found relative hypermetabolism in the SMA and precentral gyrus in $A D$, which is in line with increased FC between the SMA and M1 cortex with the sensorimotor, cingulate, and fusiform cortices both in $\mathrm{MCl}$ and $\mathrm{AD}$ patients $[53,54]$. As amyloid and tau accumulation in the $\mathrm{M} 1$ and SMA has do not occur until late stages of $A D$ [55-57], we speculate that the observed SMA relative hypermetabolism could be a compensatory phenomenon, rather than a result of direct AD pathology. Nonetheless, as regional brain FDG uptakes are normalized for global brain metabolism in our study, this observation might be a relative phenomenon. Put differently, as glucose uptake reduces in several brain regions, areas like the motor cortices with preserved metabolism appear to show a higher rFDG. Conversely, we observed reduced both $C C$ and $D C$ in the precentral gyrus and SMA in $\mathrm{MCl}$ and $A D$, indicating lower local and global information processing in the functional connectome of the SMA. Beside the motor areas, we observed rFDG and FC topology alterations in the lateral occipital cortex and occipital fusiform gyrus, which play a key role in visuospatial disturbance in patients with $A D[58,59](S I-$ Tables 1, 2 and 3). We assume that tau pathology might be responsible for rFDG and topology alterations beyond the $\mathrm{DMN}$. This is perhaps supported by the fact that tau deposition might be more strongly associated with rFDG decline, than amyloid deposition [60]. Moreover, connectivity analysis based on tau imaging showed moderate spatial overlap, not only within the DMN, but also in the visual and language networks [61].

In addition, we investigated another hypothesis considering $\mathrm{CC} / \mathrm{DC}$ and clinical diagnosis as the predictive factors and investigated whether their interaction could predict rFDG aletrations. This analysis 
revealed a significant interaction between clinical diagnosis as AD and CC/DC in left supramarginal, left middle temporal gyri, and left posterior parahippocampal gyrus, which are part of the medial temporal lobe and further highlights the role of DMN in pathophysiology of AD. In line with our findings, the supramarginal gyrus has shown reduced inter-regional FC, as well as loss of long-range FC with the central executive and frontoparietal network in $A D$ [62]. While regional hypometabolism in the supramarginal gyrus is shown to have a low discriminative value for AD [63] and changes in rFDG where not shown to be significant between $A D$ and HCs in our study, the interaction between clinical diagnosis as $A D$ and rFDG was able to predict changes in CC of the supramarginal gyrus. The parahippocampal gyrus is also part of the medial temporal lobe, which has shown in inverse $U$ pattern of change in local metabolism along $A D$ trajectory [64]. It has been hypothesized that amyloid beta accumulation in the posteromedial cortex disrupts its long-range FC with the medical temporal lobe, which in turns gives rise to disinhibition and increased local metabolism in the medical temporal lobe including the parahippocampal gyrus. These changes enhance regional amyloid and tau deposition in the temporal lobe, further FC disruption to the remote areas, and finally widespread atrophy. Our findings are in line with this hypothesis, as we observed that $A D$ interacts with loss of $D C$ to predict regional relative hypermetabolism in medical temporal lobe regions including the parahippocampal gyrus [5].

\subsection{Strengths and limitations}

A major strength of the current study was simultaneous PET/MR acquisition, which measures both FDGPET and rs-fMRI at the same time, which provides a great opportunity to test the moderating effect of disease on the association between rFDG and FC topology. An important drawback of the current study, however, was the absence of amyloid and tau imaging, which could help to assess the credibility of our hypothesis regarding the role of AD pathology on rFDG and FC topology coupling. Thus, we find it imperative for future studies to test the pathogenic role of amyloid/tau on metabolism-topology decoupling through simultaneous in vivo amyloid and tau PET imaging. Another limitation was the age gap between patients and HCs. Although we corrected for the effect of age by adding it as covariate of no-interest in our GLM models, the residual effect of age may still exist or has not-linear effects on functional brain networks [65]. We also find it compelling to validate the current results using larger datasets. Other potential limitation of this study is the cross-sectional design, which could impede the drawing of a causal relationship between rFDG and topology changes in AD.

\section{Conclusion}

The present study using simultaneous evaluation of FDG-PET and rs-fMRI provides evidence regarding regional neural activity and inter-regional $\mathrm{FC}$ alterations in $\mathrm{MCl}$ and $\mathrm{AD}$ and extends previous findings of aberrant interaction between them. We demonstrated not only regional metabolism and inter-regional FC were disrupted in patients with $\mathrm{MCl}$ and $A D$, but also there is an adverse effect of $A D$ on coupling between regional metabolism and inter-regional FC, particularly in the right ITG and SMA, as well as left middle temporal, supramarginal, and parahippocampal regions. Put differently, abnormal glucose uptake is linked to aberrant communication among brain regions, probably due to the underlying pathological 
processes in AD. However, future longitudinal studies with larger sample size should further test the role of pathological biomarkers of $A D$ (e.g., amyloid and tau proteins) on the interplay between regional metabolism and functional dysconnectivity along the trajectory of disease.

\section{Declarations}

\section{Acknowledgements}

We would like to thank all subjects for their participation in the study. Furthermore, the authors thank the staff of the Departments of Psychiatry and Psychotherapy and also Nuclear Medicine of Klinikum rechts der Isar for their help in recruitment and data collection.

\section{Funding}

No funding is related to this manuscript.

\section{Availability of data and material (data transparency)}

The datasets generated during and/or analysed during the current study are not publicly available due to [REASON(S) WHY DATA ARE NOT PUBLIC] but are available from the corresponding author on reasonable request.

\section{Code availability}

Code can be shared upon reasonable request from the corresponding author.

\section{Ethics approval}

This study was approved by the TUM ethics committee in line with the institute's Human Research Committee guidelines and conformed to standards of the declaration of Helsinki.

\section{Consent to participate}

Written informed consent was obtained from all participants after providing detailed information about this study.

\section{Conflict of interest}

The authors declare that the research was conducted in the absence of any commercial or financial relationships that could be construed as a potential conflict of interest.

\section{References}

1. Minoshima S, Frey KA, Koeppe RA, Foster NL, Kuhl DE. A diagnostic approach in Alzheimer's disease using three-dimensional stereotactic surface projections of fluorine-18-FDG PET. J Nucl Med. 
1995;36:1238-48.

2. Chetelat G, Desgranges B, Landeau B, Mezenge F, Poline JB, de la Sayette V, et al. Direct voxel-based comparison between grey matter hypometabolism and atrophy in Alzheimer's disease. Brain. 2008;131:60-71. doi:10.1093/brain/awm288.

3. Drzezga A, Grimmer T, Henriksen G, Stangier I, Perneczky R, Diehl-Schmid J, et al. Imaging of amyloid plaques and cerebral glucose metabolism in semantic dementia and Alzheimer's disease.

Neurolmage. 2008;39:619-33. doi:10.1016/j.neuroimage.2007.09.020.

4. Sorg C, Riedl V, Muhlau M, Calhoun VD, Eichele T, Laer L, et al. Selective changes of resting-state networks in individuals at risk for Alzheimer's disease. Proc Natl Acad Sci USA. 2007;104:18760-5. doi:10.1073/pnas.0708803104.

5. Pasquini L, Rahmani F, Maleki-Balajoo S, La Joie R, Zarei M, Sorg C, et al. Medial Temporal Lobe Disconnection and Hyperexcitability Across Alzheimer's Disease Stages. J Alzheimers Dis Rep. 2019;3:103-12. doi:10.3233/ADR-190121.

6. Tahmasian M, Pasquini L, Scherr M, Meng C, Forster S, Mulej Bratec S, et al. The lower hippocampus global connectivity, the higher its local metabolism in Alzheimer disease. Neurology. 2015;84:195663. doi:10.1212/WNL.0000000000001575.

7. Rubinov M, Sporns 0 . Complex network measures of brain connectivity: uses and interpretations. Neurolmage. 2010;52:1059-69. doi:10.1016/j.neuroimage.2009.10.003.

8. Masuda N, Sakaki M, Ezaki T, Watanabe T. Clustering Coefficients for Correlation Networks. Frontiers in Neuroinformatics. 2018;12. doi:10.3389/fninf.2018.00007.

9. Attwell D, Laughlin SB. An energy budget for signaling in the grey matter of the brain. Journal of cerebral blood flow metabolism: official journal of the International Society of Cerebral Blood Flow Metabolism. 2001;21:1133-45. doi:10.1097/00004647-200110000-00001.

10. Hipp JF, Hawellek DJ, Corbetta M, Siegel M, Engel AK. Large-scale cortical correlation structure of spontaneous oscillatory activity. Nat Neurosci. 2012;15:884-90. doi:10.1038/nn.3101.

11. Riedl V, Bienkowska K, Strobel C, Tahmasian M, Grimmer T, Forster S, et al. Local Activity Determines Functional Connectivity in the Resting Human Brain: A Simultaneous FDG-PET/fMRI Study. The Journal of neuroscience: the official journal of the Society for Neuroscience. 2014;34:6260-6. doi:10.1523/JNEUROSCI.0492-14.2014.

12. Savio A, Fünger $S$, Tahmasian M, Rachakonda S, Manoliu A, Sorg C, et al. Resting-State Networks as Simultaneously Measured with Functional MRI and PET. J Nucl Med. 2017;58:1314-7. doi:10.2967/jnumed.116.185835.

13. Jamadar SD, Ward PGD, Close TG, Fornito A, Premaratne M, O'Brien K, et al. Simultaneous BOLDfMRI and constant infusion FDG-PET data of the resting human brain. Sci Data. 2020;7:363. doi:10.1038/s41597-020-00699-5.

14. Tomasi D, Wang G-J, Volkow ND. Energetic cost of brain functional connectivity. Proceedings of the National Academy of Sciences. 2013;110:13642. doi:10.1073/pnas.1303346110. 
15. Marchitelli R, Aiello M, Cachia A, Quarantelli M, Cavaliere C, Postiglione A, et al. Simultaneous restingstate FDG-PET/fMRI in Alzheimer Disease: Relationship between glucose metabolism and intrinsic activity. Neurolmage. 2018;176:246-58. doi:10.1016/j.neuroimage.2018.04.048.

16. Scherr M, Pasquini L, Benson G, Nuttall R, Gruber M, Neitzel J, et al. Decoupling of Local Metabolic Activity and Functional Connectivity Links to Amyloid in Alzheimer's Disease. Journal of Alzheimer's disease: JAD. 2018;64:405-15. doi:10.3233/jad-180022.

17. Scherr M, Utz L, Tahmasian M, Pasquini L, Grothe MJ, Rauschecker JP, et al. Effective connectivity in the default mode network is distinctively disrupted in Alzheimer's disease-A simultaneous restingstate FDG-PET/fMRI study. Hum Brain Mapp. 2019. doi:10.1002/hbm.24517.

18. Tahmasian M, Shao J, Meng C, Grimmer T, Diehl-Schmid J, Yousefi BH, et al. Based on the Network Degeneration Hypothesis: Separating Individual Patients with Different Neurodegenerative Syndromes in a Preliminary Hybrid PET/MR Study. J Nucl Med. 2016;57:410-5. doi:10.2967/jnumed.115.165464.

19. Keuken MC, Bazin PL, Crown L, Hootsmans J, Laufer A, Muller-Axt C, et al. Quantifying interindividual anatomical variability in the subcortex using 7 T structural MRI. Neurolmage. 2014;94:406. doi:10.1016/j.neuroimage.2014.03.032.

20. Cranston I, Reed LJ, Marsden PK, Amiel SA. Changes in regional brain (18)F-fluorodeoxyglucose uptake at hypoglycemia in type 1 diabetic men associated with hypoglycemia unawareness and counter-regulatory failure. Diabetes. 2001;50:2329-36.

21. Bullmore E, Sporns O. The economy of brain network organization. Nat Rev Neurosci. 2012;13:33649. doi:10.1038/nrn3214.

22. Kaiser M. A tutorial in connectome analysis: topological and spatial features of brain networks. Neurolmage. 2011;57:892-907. doi:10.1016/j.neuroimage.2011.05.025.

23. van Wijk BCMS, Daffertshofer CJ. A. Comparing Brain Networks of Different Size and Connectivity Density Using Graph Theory. PLoS ONE. 2010;5:e13701. doi:10.1371/journal.pone.0013701.

24. Sadeghi M, Khosrowabadi R, Bakouie F, Mahdavi H, Eslahchi C, Pouretemad H. Screening of autism based on task-free fMRI using graph theoretical approach. Psychiatry Res Neuroimaging. 2017;263:48-56. doi:10.1016/j.pscychresns.2017.02.004.

25. van den Heuvel MP, Hulshoff Pol HE. Exploring the brain network: a review on resting-state fMRI functional connectivity. Eur Neuropsychopharmacol. 2010;20:519-34. doi:10.1016/j.euroneuro.2010.03.008.

26. Sporns 0 . Network attributes for segregation and integration in the human brain. Curr Opin Neurobiol. 2013;23:162-71. doi:10.1016/j.conb.2012.11.015.

27. Gong G, Rosa-Neto P, Carbonell F, Chen ZJ, He Y, Evans AC. Age- and gender-related differences in the cortical anatomical network. The Journal of neuroscience: the official journal of the Society for Neuroscience. 2009;29:15684-93. doi:10.1523/JNEUROSCI.2308-09.2009.

28. Meng C, Brandl F, Tahmasian M, Shao J, Manoliu A, Scherr M, et al. Aberrant topology of striatum's connectivity is associated with the number of episodes in depression. Brain. 2014;137:598-609. 
doi:10.1093/brain/awt290.

29. Aiken LS, West SG. Multiple regression: Testing and interpreting interactions. Thousand Oaks: Sage Publications, Inc.; 1991.

30. Whisman MA, McClelland GH. Designing, testing, and interpreting interactions and moderator effects in family research. J Fam Psychol. 2005;19:111-20. doi:10.1037/0893-3200.19.1.111.

31. Mosconi L, Mistur R, Switalski R, Tsui WH, Glodzik L, Li Y, et al. FDG-PET changes in brain glucose metabolism from normal cognition to pathologically verified Alzheimer's disease. Eur J Nucl Med Mol Imaging. 2009;36:811-22. doi:10.1007/s00259-008-1039-z.

32. Pasquini L, Scherr M, Tahmasian M, Meng C, Myers NE, Ortner M, et al. Link between hippocampus' raised local and eased global intrinsic connectivity in AD. Alzheimers Dement. 2015;11:475-84. doi:10.1016/j.jalz.2014.02.007.

33. Ashraf A, Fan Z, Brooks D, Edison P. Cortical hypermetabolism in MCl subjects: a compensatory mechanism? Eur J Nucl Med Mol Imaging. 2015;42:447-58.

34. Pereira JB, Mijalkov M, Kakaei E, Mecocci P, Vellas B, Tsolaki M, et al. Disrupted Network Topology in Patients with Stable and Progressive Mild Cognitive Impairment and Alzheimer's Disease. Cerebral cortex (New York, NY: 1991). 2016;26:3476-93. doi:10.1093/cercor/bhw128.

35. Strain JF, Smith RX, Beaumont H, Roe CM, Gordon BA, Mishra S, et al. Loss of white matter integrity reflects tau accumulation in Alzheimer disease defined regions. Neurology. 2018;91:e313-e8. doi:10.1212/WNL.0000000000005864.

36. Klupp E, Grimmer T, Tahmasian M, Sorg C, Yakushev I, Yousefi BH, et al. Prefrontal hypometabolism in Alzheimer disease is related to longitudinal amyloid accumulation in remote brain regions. J Nucl Med. 2015;56:399-404. doi:10.2967/jnumed.114.149302.

37. LaFerla FM, Oddo S. Alzheimer's disease: $A \beta$, tau and synaptic dysfunction. Trends Mol Med. 2005;11:170-6.

38. Spires-Jones TL, Hyman BT. The intersection of amyloid beta and tau at synapses in Alzheimer's disease. Neuron. 2014;82:756-71.

39. Schultz AP, Chhatwal JP, Hedden T, Mormino EC, Hanseeuw BJ, Sepulcre J, et al. Phases of Hyperconnectivity and Hypoconnectivity in the Default Mode and Salience Networks Track with Amyloid and Tau in Clinically Normal Individuals. The Journal of neuroscience: the official journal of the Society for Neuroscience. 2017;37:4323-31. doi:10.1523/jneurosci.3263-16.2017.

40. Jones DT, Graff-Radford J, Lowe VJ, Wiste HJ, Gunter JL, Senjem ML, et al. Tau, amyloid, and cascading network failure across the Alzheimer's disease spectrum. Cortex; a journal devoted to the study of the nervous system and behavior. 2017;97:143-59. doi:10.1016/j.cortex.2017.09.018.

41. Scheff SW, Price DA, Schmitt FA, Scheff MA, Mufson EJ. Synaptic loss in the inferior temporal gyrus in mild cognitive impairment and Alzheimer's disease. Journal of Alzheimer's disease: JAD. 2011;24:547-57. doi:10.3233/jad-2011-101782.

42. Morbelli S, Bauckneht M, Arnaldi D, Picco A, Pardini M, Brugnolo A, et al. 18F-FDG PET diagnostic and prognostic patterns do not overlap in Alzheimer's disease (AD) patients at the mild cognitive 
impairment (MCl) stage. Eur J Nucl Med Mol Imaging. 2017;44:2073-83. doi:10.1007/s00259-0173790-5.

43. Convit A, de Asis J, de Leon MJ, Tarshish CY, De Santi S, Rusinek H. Atrophy of the medial occipitotemporal, inferior, and middle temporal gyri in non-demented elderly predict decline to Alzheimer's disease. Neurobiol Aging. 2000;21:19-26.

44. Halawa OA, Gatchel JR, Amariglio RE, Rentz DM, Sperling RA, Johnson KA, et al. Inferior and medial temporal tau and cortical amyloid are associated with daily functional impairment in Alzheimer's disease. Alzheimer's research therapy. 2019;11:14. doi:10.1186/s13195-019-0471-6.

45. Weissberger GH, Melrose RJ, Fanale CM, Veliz JV, Sultzer DL. Cortical Metabolic and Cognitive Correlates of Disorientation in Alzheimer's Disease. Journal of Alzheimer's disease: JAD. 2017;60:707-19. doi:10.3233/jad-170420.

46. Tosun D, Landau S, Aisen PS, Petersen RC, Mintun M, Jagust W, et al. Association between tau deposition and antecedent amyloid-beta accumulation rates in normal and early symptomatic individuals. Brain. 2017;140:1499-512. doi:10.1093/brain/awx046.

47. Dorsaint-Pierre R, Penhune VB, Watkins KE, Neelin P, Lerch JP, Bouffard M, et al. Asymmetries of the planum temporale and Heschl's gyrus: relationship to language lateralization. Brain. 2006;129:116476. doi:10.1093/brain/awl055.

48. Hopkins WD, Nir TM. Planum temporale surface area and grey matter asymmetries in chimpanzees (Pan troglodytes): the effect of handedness and comparison with findings in humans. Behav Brain Res. 2010;208:436-43. doi:10.1016/j.bbr.2009.12.012.

49. Kutova M, Mrzilkova J, Riedlova J, Zach P. Asymmetric Changes in Limbic Cortex and Planum Temporale in Patients with Alzheimer Disease. Curr Alzheimer Res. 2018;15:1361-8. doi:10.2174/1567205015666181004142659.

50. Chance SA, Clover L, Cousijn H, Currah L, Pettingill R, Esiri MM. Microanatomical correlates of cognitive ability and decline: normal ageing, $\mathrm{MCl}$, and Alzheimer's disease. Cerebral cortex (New York, NY: 1991). 2011;21:1870-8. doi:10.1093/cercor/bhq264.

51. Brown RK, Bohnen NI, Wong KK, Minoshima S, Frey KA. Brain PET in suspected dementia: patterns of altered FDG metabolism. Radiographics: a review publication of the Radiological Society of North America Inc. 2014;34:684-701. doi:10.1148/rg.343135065.

52. Marcus C, Mena E, Subramaniam RM. Brain PET in the diagnosis of Alzheimer's disease. Clinical nuclear medicine. 2014;39:e413-e26. doi:10.1097/RLU.0000000000000547.

53. Vidoni ED, Thomas GP, Honea RA, Loskutova N, Burns JM. Evidence of altered corticomotor system connectivity in early-stage Alzheimer's disease. Journal of neurologic physical therapy: JNPT. 2012;36:8-16. doi:10.1097/NPT.0b013e3182462ea6.

54. Cai S, Chong T, Peng Y, Shen W, Li J, von Deneen KM, et al. Altered functional brain networks in amnestic mild cognitive impairment: a resting-state fMRI study. Brain imaging behavior. 2017;11:619-31. doi:10.1007/s11682-016-9539-0. 
55. Lyoo CH, Cho H, Choi JY, Hwang MS, Hong SK, Kim YJ, et al. Tau Accumulation in Primary Motor Cortex of Variant Alzheimer's Disease with Spastic Paraparesis. Journal of Alzheimer's disease: JAD. 2016;51:671-5. doi:10.3233/jad-151052.

56. Grothe MJ, Barthel H, Sepulcre J, Dyrba M, Sabri O, Teipel SJ. In vivo staging of regional amyloid deposition. Neurology. 2017;89:2031-8. doi:10.1212/wnl.0000000000004643.

57. Del Tredici K, Braak H. Spreading of Tau Pathology in Sporadic Alzheimer's Disease Along Corticocortical Top-Down Connections. Cereb Cortex. 2018;28:3372-84. doi:10.1093/cercor/bhy152.

58. Cerquera-Jaramillo MA, Nava-Mesa MO, Gonzalez-Reyes RE, Tellez-Conti C, de-la-Torre A. Visual Features in Alzheimer's Disease: From Basic Mechanisms to Clinical Overview. Neural Plast. 2018;2018:2941783. doi:10.1155/2018/2941783.

59. Brewer A, Barton B. Visual cortex in aging and Alzheimer's disease: changes in visual field maps and population receptive fields. Front Psychol. 2014;5. doi:10.3389/fpsyg.2014.00074.

60. Ossenkoppele R, Schonhaut DR, Scholl M, Lockhart SN, Ayakta N, Baker SL, et al. Tau PET patterns mirror clinical and neuroanatomical variability in Alzheimer's disease. Brain. 2016;139:1551-67. doi:10.1093/brain/aww027.

61. Hoenig MC, Bischof GN, Seemiller J, Hammes J, Kukolja J, Onur OA, et al. Networks of tau distribution in Alzheimer's disease. Brain. 2018;141:568-81. doi:10.1093/brain/awx353.

62. Zhao Q, Sang X, Metmer H, Swati Z, Lu J. Alzheimer's Disease Neurolmaging I. Functional segregation of executive control network and frontoparietal network in Alzheimer's disease. Cortex. 2019;120:36-48. doi:10.1016/j.cortex.2019.04.026.

63. Yun HJ, Kwak K, Lee J-M, Alzheimer's Disease Neuroimaging I. Multimodal Discrimination of Alzheimer's Disease Based on Regional Cortical Atrophy and Hypometabolism. PLOS ONE. 2015;10:e0129250. doi:10.1371/journal.pone.0129250.

64. Dickerson BC, Sperling RA. Functional abnormalities of the medial temporal lobe memory system in mild cognitive impairment and Alzheimer's disease: insights from functional MRI studies. Neuropsychologia. 2008;46:1624-35. doi:10.1016/j.neuropsychologia.2007.11.030.

65. Betzel RF, Byrge L, He Y, Goni J, Zuo XN, Sporns O. Changes in structural and functional connectivity among resting-state networks across the human lifespan. Neurolmage. 2014;102 Pt 2:345 - 57. doi:10.1016/j.neuroimage.2014.07.067.

\section{Figures}




\section{Regional glucose metabolism}

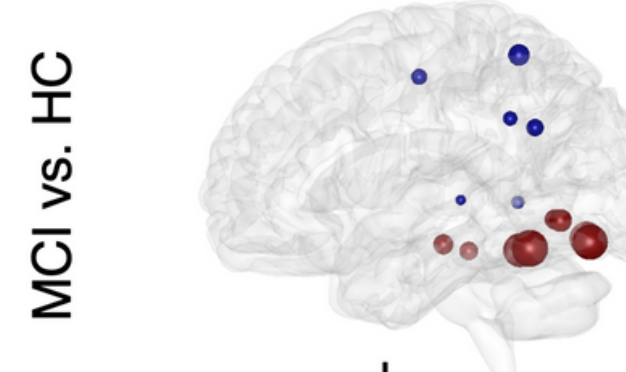

L

- Relative hypermetabolism in $\mathrm{MCl}$
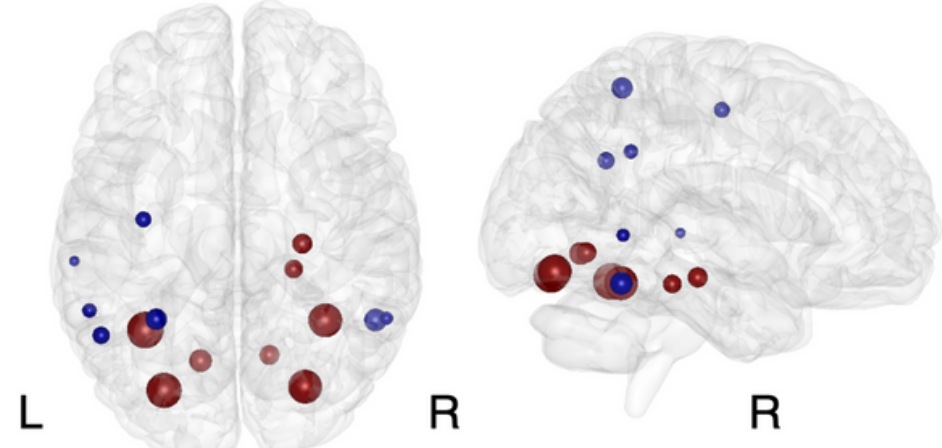

- Hypometabolism in $\mathrm{MCl}$
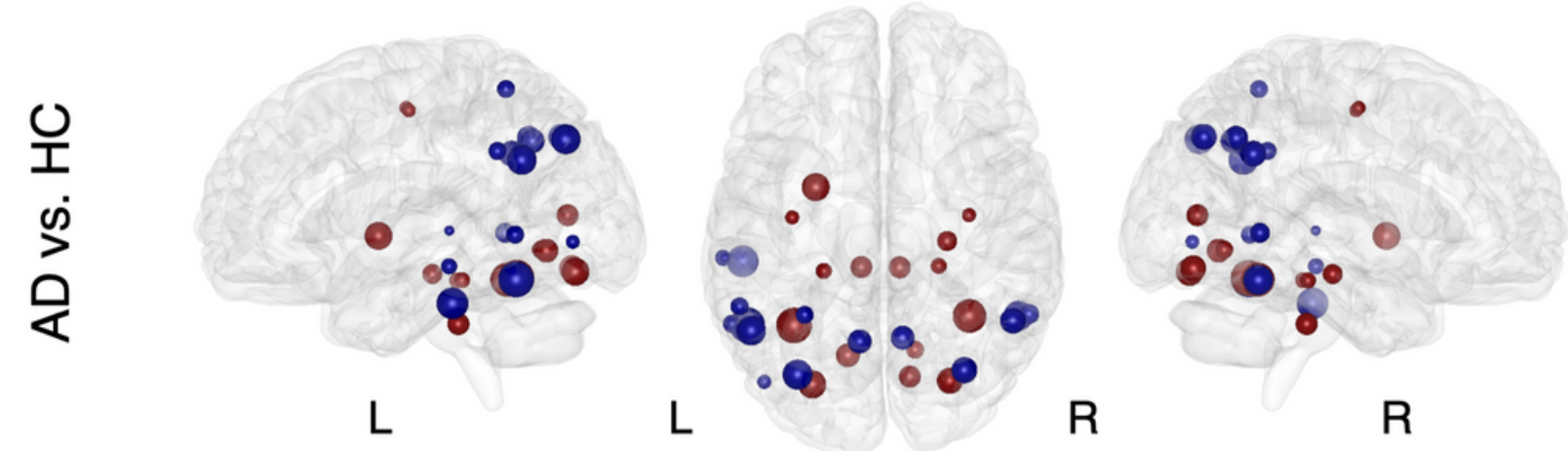

Relative hypermetabolism in AD

- Hypometabolism in AD

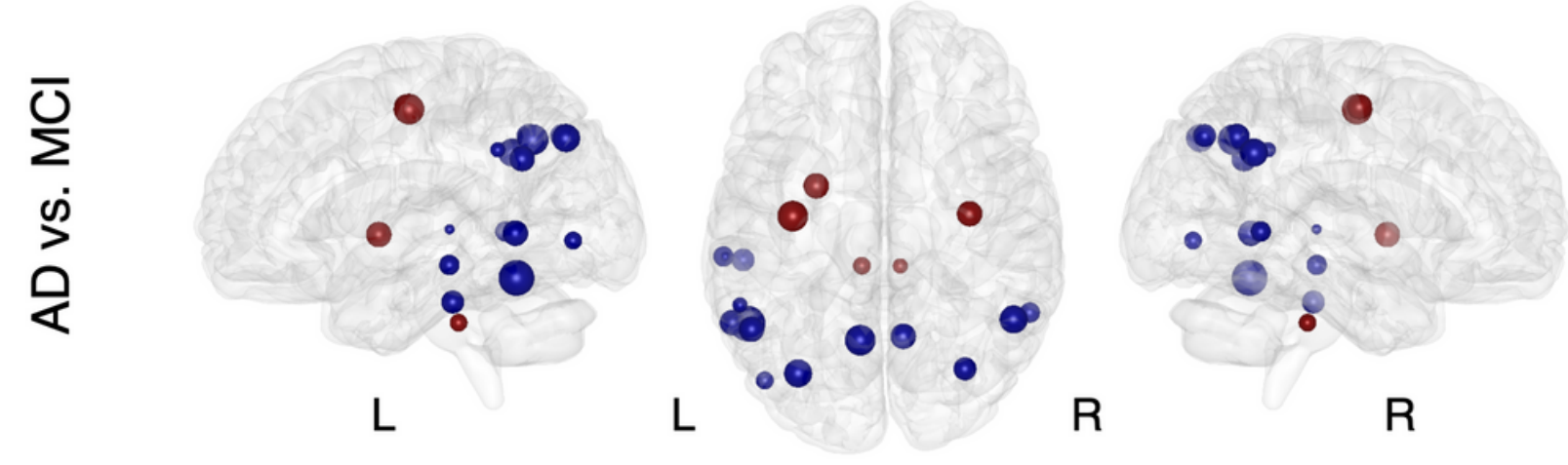

- Relative hypermetabolism in AD

- Hypometabolism in AD

\section{Figure 1}

Analysis of covariance on regional glucose metabolism for each region in Harvard-Oxford Atlas. Age and sex were considered as the covariates of no-interest. Post-hoc test: permutation test $(p<0.05,100,000$ permutations). The size of each node corresponds to absolute mean difference values of regional glucose metabolism between each pair of groups. AD: Alzheimer disease; HC: healthy control; $\mathrm{MCl}$ : mild cognitive impairment; L: left; R: right. 


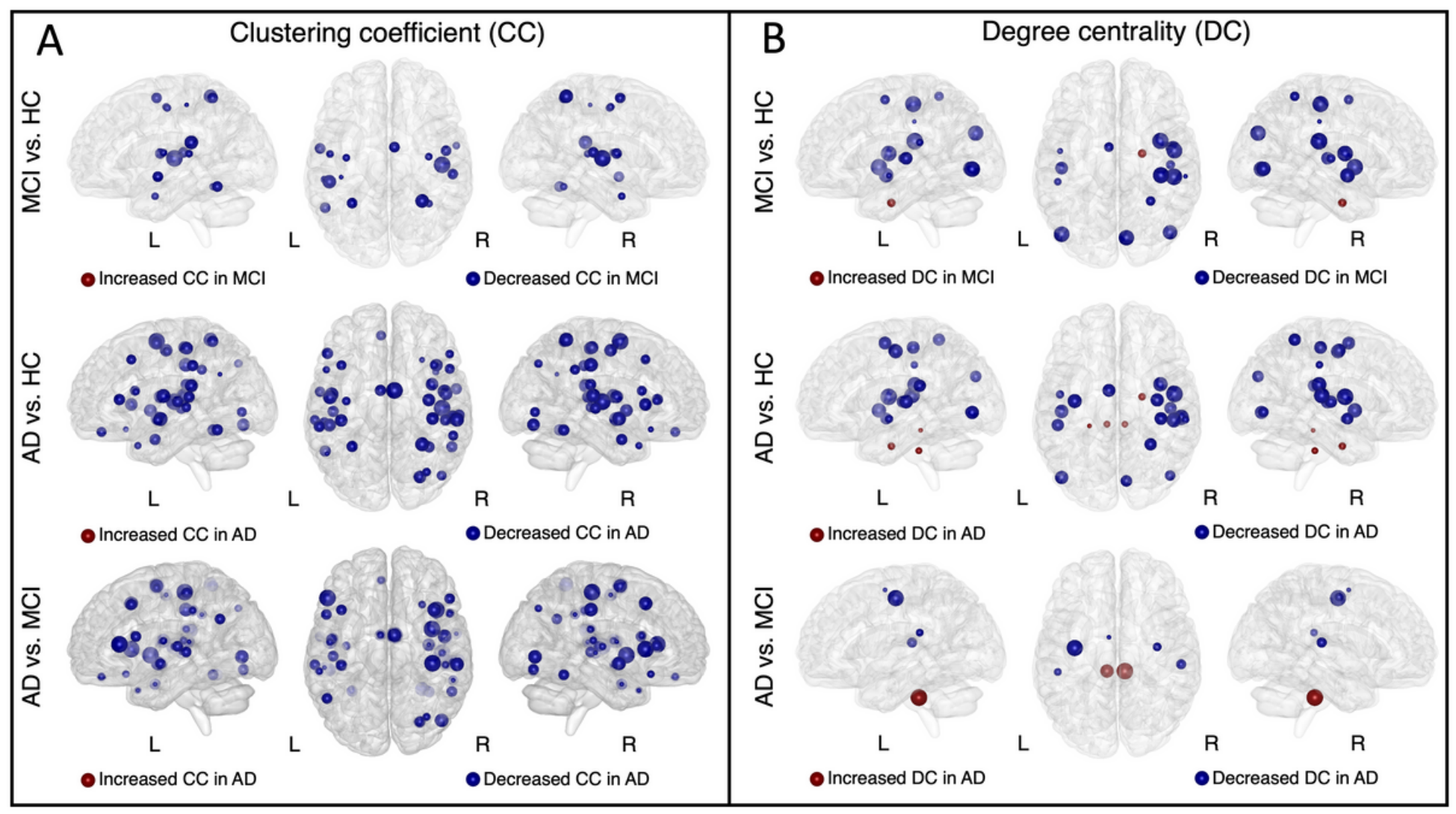

Figure 2

Analysis of covariance on whole brain FC topological metrics for each region in Harvard-0xford Atlas in A) clustering coefficient; B) degree centrality. Age and sex were considered as the covariates of nointerest. Post-hoc test: permutation test ( $p<0.05,100,000$ permutations). The size of each node corresponds to absolute mean difference values of clustering coefficient/degree centrality between each pair of groups. FC: functional connectivity; AD: Alzheimer disease; $\mathrm{HC}$ : healthy control; $\mathrm{MCl}$ : mild cognitive impairment; L: left; R: right. 


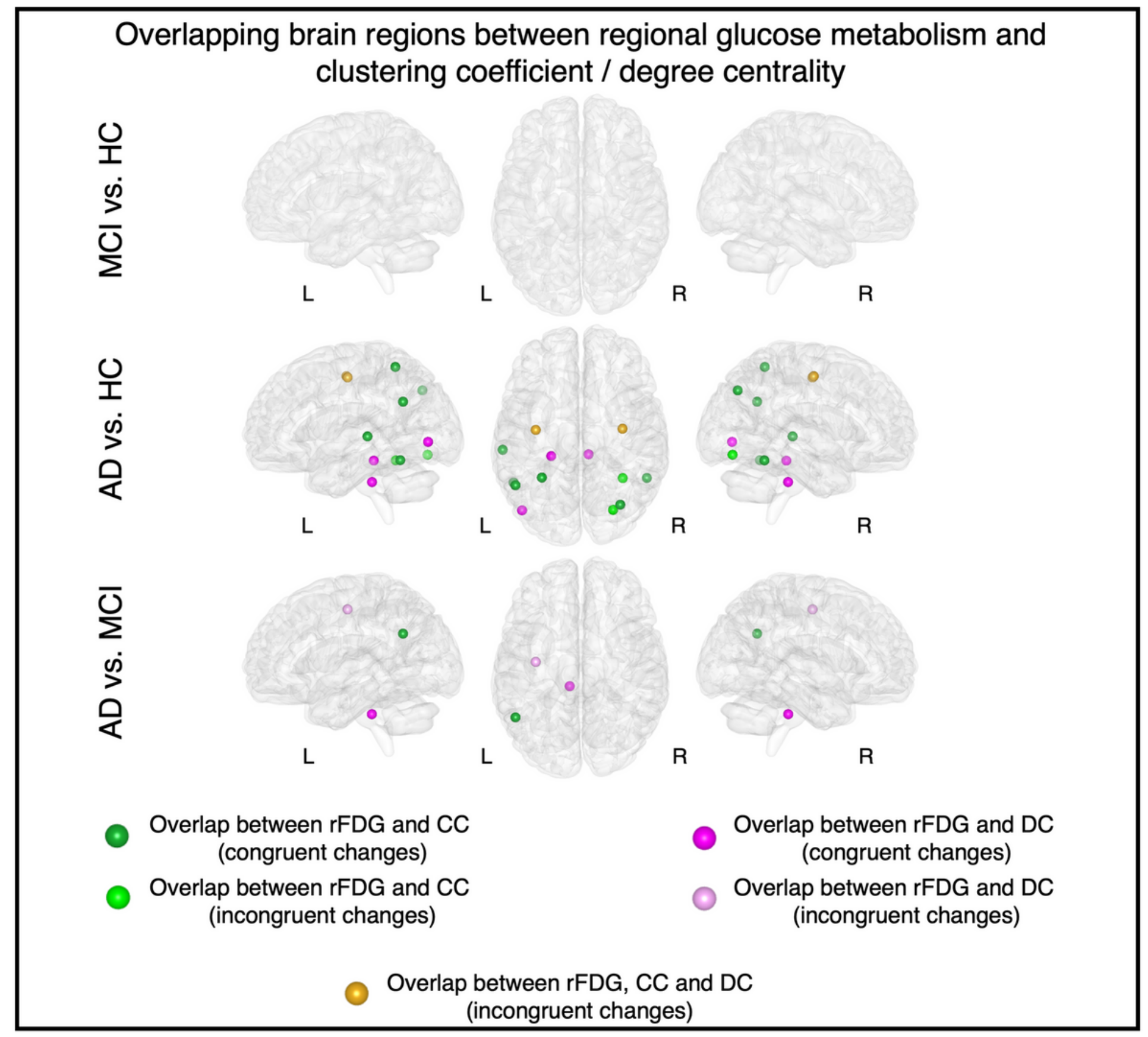

Figure 3

Overlapping brain regions between regional glucose metabolism and inter-regional FC topology metrics (clustering coefficient, and degree centrality). Congruent changes refer to similar direction of regional glucose metabolism and clustering coefficient/degree centrality between each pair of groups. rFDG: regional glucose metabolism identified through FDG-PET; CC: clustering coefficient; DC: degree centrality; FC: functional connectivity; $\mathrm{AD}$ : Alzheimer disease; $\mathrm{HC}$ : healthy control; $\mathrm{MCl}$ : mild cognitive impairment; $\mathrm{L}$ : left; R: right. 


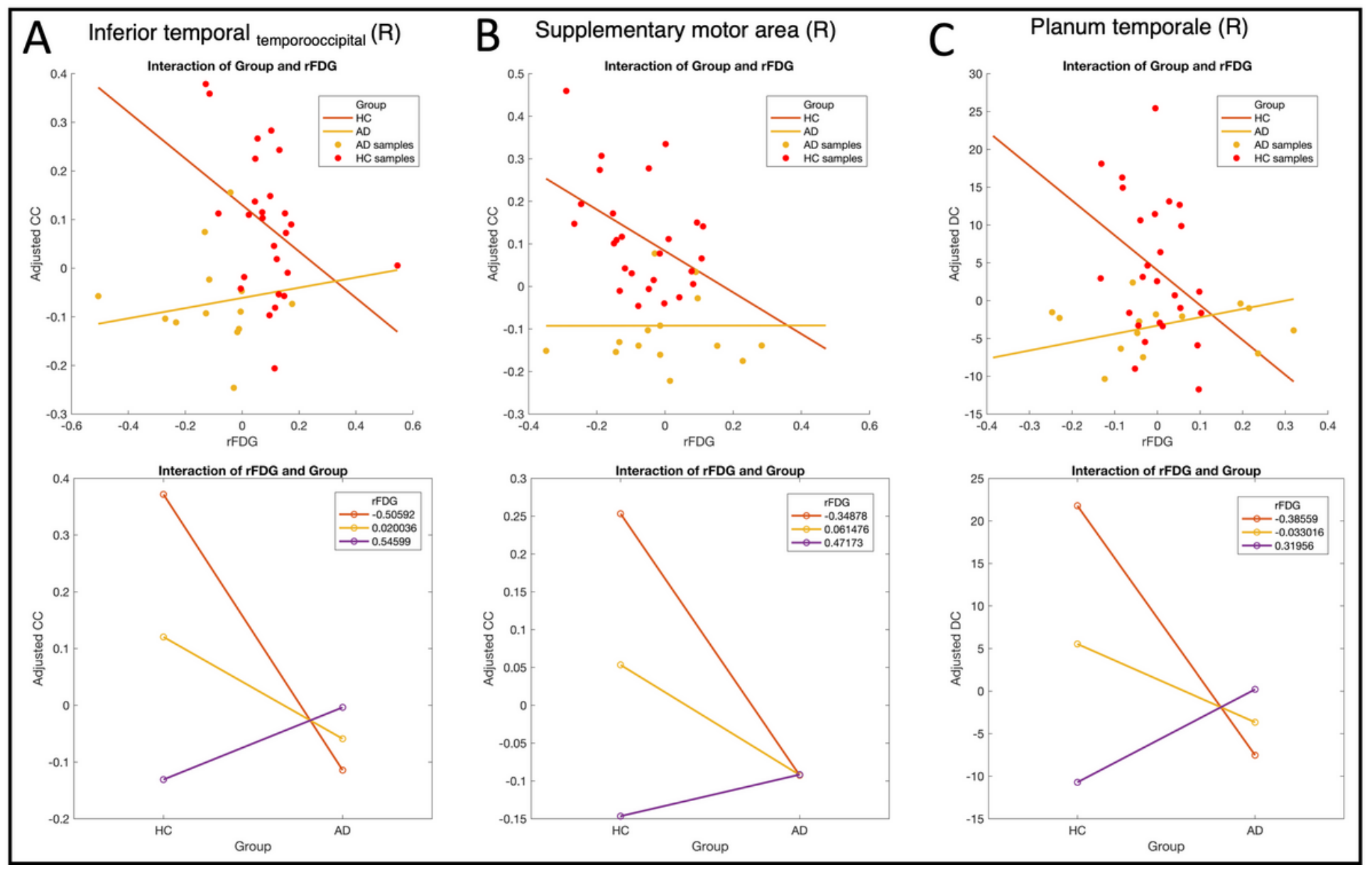

Figure 4

AD moderated the effect of regional glucose metabolism ( $\mathrm{rFDG}$ ) on predicting the $\mathrm{FC}$ derived topological measures (CC/DC) based on rFDG in overlapping/non-overlapping regions. Results demonstrated that only in A) right inferior temporal gyrus-temporooccipital part (overlapping region) and B) right supplementary motor area (non-overlapping region) defined by group comparison between $A D$ and $H C$, HMMR analysis predicted CC significantly with the two-way interaction (rFDG $\times$ clinical diagnosis). Moreover, in $\mathrm{C}$ ) right planum temporale (non-overlapping region) defined by group comparison between $A D$ and $H C, H M M R$ analysis predicted DC significantly with the two-way interaction ( $r F D G \times$ clinical diagnosis). Figures in the first row indicate that CC/DC decreases as a function of rFDG in HC subjects but increases in $A D$ patients. In the second row, we showed changes in the CC/DC in both $H C$ and $A D$ groups, when the rFDG is fixed at three low, medium and high levels. The lines for $\mathrm{HC}$ and $\mathrm{AD}$ are crossing, indicating an interaction between rFDG and clinical diagnosis. AD: Alzheimer's disease; HC: healthy control; HMMR: hierarchical moderated multiple regression; CC: clustering coefficient; DC: degree centrality.

\section{Supplementary Files}

This is a list of supplementary files associated with this preprint. Click to download. 
- SupportingInformation.docx

Page 23/23 